\title{
Monte Carlo studies of Matrix theory correlation functions
}

\author{
Masanori Hanada ${ }^{1} *$ Jun Nishimura ${ }^{2,3}$ 团 Yasuhiro Sekino 4 团 and Tamiaki Yoneya 9 \\ ${ }^{1}$ Department of Particle Physics and Astrophysics, \\ Weizmann Institute of Science, Rehovot 76100, Israel \\ ${ }^{2}$ KEK Theory Center, High Energy Accelerator Research Organization, Tsukuba 305-0801, Japan \\ ${ }^{3}$ Department of Particle and Nuclear Physics, School of High Energy Accelerator Science, \\ Graduate University for Advanced Studies (SOKENDAI), Tsukuba 305-0801, Japan \\ ${ }^{4}$ Okayama Institute for Quantum Physics, 1-9-1 Kyoyama, Okayama 700-0015, Japan \\ ${ }^{5}$ Institute of Physics, University of Tokyo, Komaba, Meguro-ku, Tokyo 153-8902, Japan
}

(Dated: November, 2009; preprint: WS/14/09/NOV-DPPA, KEK-TH-1337, OIQP-09-12, UT-Komaba/09-5)

\begin{abstract}
We study correlation functions in $(0+1)$-dimensional maximally supersymmetric $\mathrm{U}(N)$ gauge theory, which represents the low-energy effective theory of D0-branes. In the large- $N$ limit, the gauge-gravity duality predicts power-law behaviors in the infrared region for the two-point correlation functions of operators corresponding to supergravity modes. We evaluate such correlation functions on the gauge theory side by the Monte Carlo method. Clear power-law behaviors are observed at $N=3$, and the predicted exponents are confirmed consistently. Our results suggest that the agreement extends to the M-theory regime, where the supergravity analysis in 10 dimensions may not be justified a priori.
\end{abstract}

PACS numbers: 11.25.-w; 11.25.Sq

Introduction.- Maximally supersymmetric YangMills theories (SYM) in various dimensions are important because of their connection to non-perturbative formulations of string theory. Of particular interest is the $(0+1)$ D SYM with $U(N)$ gauge symmetry, which is supposed to describe the low-energy gravitational dynamics of D0-branes in 10D type IIA superstring theory. In an appropriate large- $N$ limit, this theory was proposed to be a definition of M-theory in a special light-like frame, and it is commonly referred to as the Matrix theory [1]. M-theory is a hypothetical 11D theory [2], whose low energy effective theory is given by $11 \mathrm{D}$ supergravity, and it is believed to appear in the strong coupling limit of $10 \mathrm{D}$ type IIA superstring theory.

Indeed it was confirmed that scattering amplitudes in the Matrix theory at weak coupling are consistent with predictions from 11D supergravity. Even the three-body force, which is a characteristic non-linear effect of general relativity, has been reproduced [3] from the quantum loop effects of massive open strings connecting D0-branes. On the other hand, direct perturbative calculations of correlation functions, which could provide crucial information on the as yet mysterious theory, are plagued by severe infrared divergences caused by the massless modes inherent in the theory. We therefore need genuinely nonperturbative methods to study such quantities.

In the case of $\mathcal{N}=4 \mathrm{SYM}$ in $(3+1)$ dimensions, various useful insights have been gained from the AdS/CFT correspondence [4, 5]. This conjectural duality enables us to study the $\mathcal{N}=4 \mathrm{SYM}$ in the large- $N$ limit with large 't Hooft coupling constant by using the weakly coupled supergravity, which describes the low-energy limit of the string theory. Likewise, SYM in $(p+1)$-dimensions, corresponding to the world-volume theory of $\mathrm{D} p$-branes, is expected to be dual to a superstring theory on the $\mathrm{D} p$ - brane background in the near-horizon limit $[\underline{6}, 7]$.

Assuming this duality for the $p=0$ case and using the known dictionary [8] between the supergravity modes and the Matrix theory operators, one may hope to calculate the correlation functions in the large- $N$ limit, on the basis of the Gubser-Klebanov-Polyakov-Witten prescription [5]. This program was carried out a decade ago by Y.S. and T.Y. 9] using the "generalized conformal symmetry" [7, 10] as a guide for classifying and interpreting the obtained results. The prediction is that the two-point correlation functions obey the power law

$$
\left\langle\mathcal{O}(t) \mathcal{O}\left(t^{\prime}\right)\right\rangle \propto \frac{1}{\left|t-t^{\prime}\right|^{2 \nu+1}},
$$

for a set of operators $\mathcal{O}(t)$ corresponding to the supergravity modes. The exponents $\nu$ are fractional numbers which differ from the canonical values, and they are related to the generalized conformal dimensions $\Delta$ of the operators under consideration by $\Delta=-1+\frac{10}{7} \nu[9]$. The power-law behavior conforms to the existence of a unique threshold bound state at zero energy [11] in the gauge theory, which corresponds to single-body states in the graviton supermultiplet.

This result has been re-derived in later works 12], extending the analysis in Ref. [9] to general D $p$-branes $(p<$ $5)$ in the PP-wave limit. There it has also been shown that the infrared behavior of the correlation functions for operators corresponding to excited stringy modes is typically $\exp \left\{-c\left(g_{\mathrm{YM}}^{2} N\left|t-t^{\prime}\right|^{3}\right)^{1 / 5}\right\}$ up to power corrections.

The near-horizon limit of the D0-brane background is valid when the radial coordinate $r$ in the bulk supergravity satisfies $r \ll\left(g_{s} N\right)^{1 / 7}$ in the string unit $\alpha^{\prime}=1$. The analysis using the supergravity approximation is then possible when $g_{s} N \gg 1$, and is trustable when both the background curvature and the effective string cou- 
pling given by the dilaton expectation value are small. The string coupling becomes stronger towards the center, while the curvature becomes larger towards the boundary. Taking into account the fact that the radial coordinate $r$ is related to the time scale $t$ at the boundary as $r \sim\left(t / \lambda^{1 / 2}\right)^{-2 / 5}[9]$ with $\lambda \equiv g_{\mathrm{YM}}^{2} N \sim g_{s} N$, the conditions for justifying the supergravity result (1) can be summarized as [6, 7, 9 ]

$$
\lambda^{-1 / 3} \ll\left|t-t^{\prime}\right| \ll \lambda^{-1 / 3} N^{10 / 21} .
$$

Hence, the application of the gauge-gravity correspondence is legitimate when one studies the region which is sufficiently infrared compared to the length scale set by $\lambda$. Since the upper bound in (2) diverges at large $N$, the infrared behavior of the correlation functions can be predicted reliably from the supergravity analysis in the large- $N$ limit.

In this Letter we evaluate the correlation functions on the gauge theory side by the Monte Carlo method [13] and compare the results with the predictions from the gauge-gravity correspondence. Related Monte Carlo analyses have been recently applied by the authors including M.H. and J.N. to reproduce the black hole thermodynamics [14, 15] and the Schwarzschild radius [17] of the dual geometry. To the best of our knowledge, this is the first attempt to compute the Matrix theory correlation functions from first principles [18]. Our Monte Carlo data are consistent with the supergravity predictions with reasonable accuracy despite the fact that the matrix size is as small as $N=3$. Possible interpretations shall be given later.

Simulating Matrix theory. - The action of the Matrix theory can be written (in the Euclidean convention) as

$$
\begin{aligned}
S= & \frac{N}{\lambda} \int_{0}^{\beta} d t \operatorname{Tr}\left\{\frac{1}{2}\left(D_{t} X_{i}\right)^{2}-\frac{1}{4}\left[X_{i}, X_{j}\right]^{2}\right. \\
& \left.+\frac{1}{2} \psi_{\alpha} D_{t} \psi_{\alpha}-\frac{1}{2} \psi_{\alpha}\left(\gamma_{i}\right)_{\alpha \beta}\left[X_{i}, \psi_{\beta}\right]\right\},
\end{aligned}
$$

where $X_{i}(i=1, \cdots, 9)$ and $\psi_{\alpha}(\alpha=1, \cdots, 16)$ are $N \times N$ bosonic and fermionic Hermitian matrices, on which the covariant derivative $D_{t}$ acts as $D_{t}=\partial_{t}-i[A, \cdot]$ with the gauge field $A$. The $16 \times 16$ matrices $\gamma_{i}$ satisfy the Clifford algebra $\left\{\gamma_{i}, \gamma_{j}\right\}=2 \delta_{i j}$. It is convenient to adopt units in which $\lambda=1$ without loss of generality. This does not contradict the conditions for the bulk theory, since the gauge theory has no independent length scale other than the coupling constant $g_{\mathrm{YM}}^{-2 / 3}$ and hence the strong coupling limit amounts to the IR limit.

In order to put the system on a computer, we have to introduce the UV and IR cutoffs appropriately. The extent $\beta$ in the Euclidean time direction represents the IR cutoff, which should be sufficiently large in order to see the correct infrared properties. Since we are not interested in the finite temperature behaviors unlike in Refs. [14, 17], we impose periodic boundary conditions for both bosonic and fermionic matrices, respecting supersymmetry. In order to introduce the UV cutoff, we first fix the gauge as $A(t)=\frac{1}{\beta} \operatorname{diag}\left(\alpha_{1}, \cdots, \alpha_{N}\right)$, where $-\pi<\alpha_{i} \leq \pi$, and then introduce a Fourier mode cutoff $\Lambda$ as $X_{i}(t)=$ $\sum_{n=-\Lambda}^{\Lambda} \tilde{X}_{i n} e^{i \omega n t}$ and $\psi_{\alpha}(t)=\sum_{n=-\Lambda}^{\Lambda} \tilde{\psi}_{\alpha n} e^{i \omega n t}$, where $\omega=2 \pi / \beta$. Integration over the fermionic matrices yields a Pfaffian, which could be complex in general. In this work, we simply neglect its phase following the argument in Ref. [17]. While we do not have purely theoretical justification of our assumption at this moment, the previous works [14, 17] provide empirical evidence that the phase does not affect the results in the continuum limit. Including its effect is possible in principle by the so-called reweighting method, but it is not feasible for our purpose, since the demand on the statistics would increase exponentially with the system size due to huge cancellation. This is generally called the "sign problem". The system of finite degrees of freedom obtained under the above assumption can be simulated by using the Rational Hybrid Monte Carlo algorithm [20] as described in Ref. [14].

Correlation functions. - First let us study a series of operators $J_{l, i_{1}, \cdots, i_{l}}^{+i j}(l \geq 1)$, which is defined by

$$
J_{l, i_{1}, \cdots, i_{l}}^{+i j} \equiv \frac{1}{N} \operatorname{Str}\left(F_{i j} X_{i_{1}} \cdots X_{i_{l}}\right)
$$

where $F_{i j} \equiv-i\left[X_{i}, X_{j}\right]$ and Str represents the symmetrized trace treating $F_{i j}$ as a single unit. The value of $\nu$ in (11) predicted in this case is $\nu=2 l / 5$ [9].

Since we are dealing with the Fourier modes in our simulation, the two-point functions which are directly accessible are those in the momentum space $\langle\tilde{\mathcal{O}}(p) \tilde{\mathcal{O}}(-p)\rangle$, where $p=n \omega$. Correlation functions in the real space can be obtained by the inverse Fourier transformation $\langle\mathcal{O}(t) \mathcal{O}(0)\rangle=\frac{1}{\beta} \sum_{p}\langle\tilde{\mathcal{O}}(p) \tilde{\mathcal{O}}(-p)\rangle e^{i p t}$. However, the results obtained in this way oscillates with the frequency of $\mathrm{O}(\Lambda \omega)$ as a result of the Gibbs phenomenon. For the series of operators (4), we find that the momentumspace correlator falls off as $p^{-2}$ at large $p$. Assuming that this behavior continues to infinite $p$, we extend it up to $p=1000 \omega$, and then make the inverse Fourier transformation, which removes the Gibbs phenomenon completely [13]. In Fig. 11, we show the real-space correlation functions obtained in this way. The predicted power-law behavior can be seen in the region $0.5 \lesssim t \lesssim 1.5$, which is somewhat wider than the criterion (2) for justifying the supergravity analysis. Note that the IR bound of (2) is $\left|t-t^{\prime}\right| \ll 1.69$ for $N=3$. Considering that 1.5 is close to $\beta / 2=2$, we expect that the deviation observed at $t \gtrsim 1.5$ is rather due to the IR cutoff.

Let us next study the operator $T_{2, j k}^{+i}$ defined by

$$
T_{2, j k}^{+i} \equiv \frac{1}{N} \operatorname{Str}\left(\left(D_{t} X_{i}\right) X_{j} X_{k}\right),
$$

for which $\nu$ is predicted to be $\nu=4 / 5$ [9]. Unlike the previous case, the two-point function in the Fourier space 


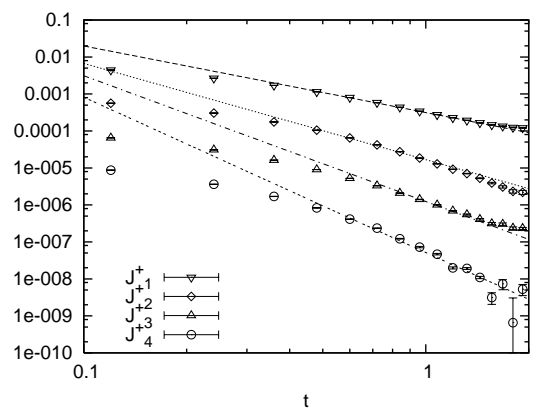

FIG. 1: The real-space two-point correlation functions $\left\langle J_{l}^{+}(t) J_{l}^{+}(0)\right\rangle(l=1,2,3,4)$ are plotted for $N=3$ and $\beta=4$. The UV cutoff is $\Lambda=16$. The straight lines are fits to the predicted power-law behavior.

does not fall off as $p^{-2}$ at large $p$. This is likely due to the presence of a derivative $D_{t}$ in (5). Furthermore, we observe considerable dependence on the UV cutoff $\Lambda$. We therefore evaluate the two-point function in the momentum space at various $\Lambda$ and make an extrapolation to $\Lambda=\infty$ at each $p$. The leading finite $\Lambda$ effects are consistent with $1 / \Lambda$ as one might expect theoretically, and we make an extrapolation with $\Lambda=6,8,12$ for $0 \leq$ $p /(2 \pi) \leq 0.6$ and $\Lambda=8,12,16$ for $p /(2 \pi) \geq 0.8$. Figure 2 shows the results obtained in this way for $N=3, \beta=5$.

On the other hand, the prediction (11) in the Fourier space reads [9]

$$
\langle\tilde{\mathcal{O}}(p) \tilde{\mathcal{O}}(-p)\rangle \simeq f(p)+|p|^{2 \nu} g(p)
$$

at small $p$, where $f(p)$ and $g(p)$ are analytic functions $(g(0) \neq 0)$, which cannot be determined by the supergravity analysis. The odd powers of $p$ in $f(p)$ and $g(p)$ are forbidden by the time reflection invariance. For the present operator, considering that $\nu=4 / 5$, a few leading terms at small $p$ are predicted to be of the form

$$
\left\langle\tilde{T}_{2}^{+}(p) \tilde{T}_{2}^{+}(-p)\right\rangle \simeq a+b p^{2}+c|p|^{2 \nu},
$$

where $a, b$ and $c$ are constants. Note that the polynomial terms do not affect the long-distance behavior of the correlation function. Treating $\nu$ as a free parameter and fitting our data in $0 \leq p /(2 \pi) \leq 1$ to (7), we obtain $\nu=0.80 \pm 0.03$ as in Fig. 2 ,

As the last example, let us consider the operator

$$
T_{2, i j}^{++} \equiv \frac{1}{N} \operatorname{tr}\left(X_{i} X_{j}\right) \quad(i \neq j)
$$

for which the supergravity analysis predicts $\nu=-3 / 5$ 9]. The two-point function is predicted to behave as $\left|t-t^{\prime}\right|^{1 / 5}$ in the real space, which is divergent as $\left|t-t^{\prime}\right| \rightarrow$ $\infty$. This IR divergence is reminiscent of the free-field behavior $\left|t-t^{\prime}\right|^{2}$ albeit with much weaker power. Here

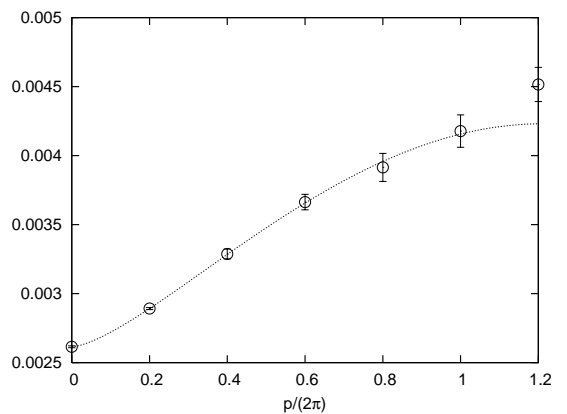

FIG. 2: The momentum-space two-point correlation function $\left\langle\tilde{T}_{2}^{+}(p) \tilde{T}_{2}^{+}(-p)\right\rangle$ is plotted for $N=3$ and $\beta=5$. The dotted line represents a fit to the behavior (7) treating $\nu$ as a free parameter in the range $0 \leq p /(2 \pi) \leq 1$. The best fit is obtained for $\nu=0.80 \pm 0.03$.

we examine the behavior (6) for small $p$ in the Fourier space. Considering that $\nu=-3 / 5$, a few leading terms are predicted to be of the form

$$
\left\langle\tilde{T}_{2}^{++}(p) \tilde{T}_{2}^{++}(-p)\right\rangle \simeq a|p|^{2 \nu}+b+c|p|^{2 \nu+2} .
$$

Note that the leading term is divergent as $p \rightarrow 0$, as opposed to the previous cases with vanishing behaviors. In Fig. 3, we show a log-log plot of the two-point function. We extrapolated our data to $\Lambda=\infty$ as we did for $T_{2}^{+}$. Treating $\nu$ as a free parameter and fitting our results to (9) in the region $0.3 \leq p /(2 \pi) \leq 2$, we obtain $\nu=$ $-0.61 \pm 0.02$. The deviation from the behavior (9) at small $p$ is most likely due to finite IR cutoff effects as one can see from the trends with increasing $\beta$.

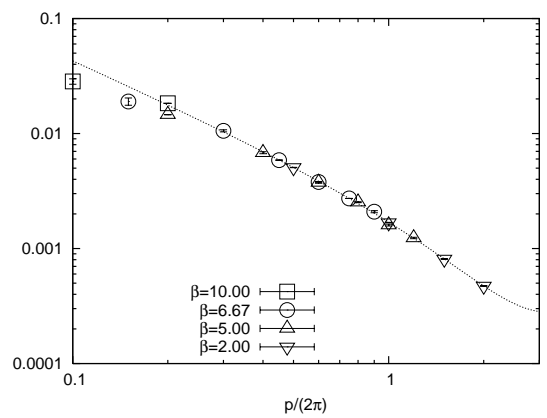

FIG. 3: The momentum-space two-point correlation function $\left\langle\tilde{T}_{2}^{++}(p) \tilde{T}_{2}^{++}(-p)\right\rangle$ is plotted in the log-log scale for $N=3$. The dotted line represents a fit to the behavior (9) treating $\nu$ as a free parameter in the range $0.3 \leq p /(2 \pi) \leq 2$. The best fit is obtained for $\nu=-0.61 \pm 0.02$.

Summary and discussion. - In this Letter we presented the first Monte Carlo evaluation of two-point correlation functions of the supergravity operators in the Matrix theory. In particular, we observed that the power 
law predicted by the gauge-gravity correspondence continues to hold in the infrared region beyond the naive criterion for the validity of the supergravity analysis.

From the gauge theory point of view, the existence of a threshold bound state at zero energy requires the power law at sufficiently large $\left|t-t^{\prime}\right|$ for any $N(\geq 2)$. In fact, the existence of a unique threshold bound state for each $N(\geq 2)$ has been argued, based on the calculation of the Witten index [21]. Let us also consider the spectral representation of the two-point function

$$
\langle\tilde{\mathcal{O}}(p) \tilde{\mathcal{O}}(-p)\rangle=\int d \mu \rho(\mu) \frac{1}{p^{2}+\mu^{2}}
$$

The power law of the two-point function at small $p$ implies that the spectral density $\rho(\mu)$ behaves as $\rho(\mu) \sim$ $\mu^{2 \nu+1}$ at small $\mu$. The fact that the exponent $\nu$ increases in general [9] with the angular momentum carried by the operator is consistent with the continuous mass spectrum of the intermediate many-body states composed of the zero-energy bound states.

On the gravity side, let us emphasize that the upper bound in (2) is merely a sufficient condition for justifying the supergravity analysis. Here we recall that the wave function of the supergravity fluctuations obtained in Ref. [9] is exponentially small in the central region. Therefore, it is possible that the 10D supergravity approximation in this region is not affected by the increasingly large effective string coupling at small $r$ and hence at large $\left|t-t^{\prime}\right|$. On the other hand, in the short-distance regime $\left|t-t^{\prime}\right| \ll \lambda^{-1 / 3}$, where the curvature becomes large on the gravity side, we expect large deviations from the powerlaw behavior (11). This is clear, in particular, for the cases with $2 \nu+1>0$ since there should be no UV singularity in one dimension. Such deviations are indeed observed in the stringy PP-wave analysis [12] on the bulk side.

Another significant aspect of our results is that the exponents obtained from the Monte Carlo data at $N=3$ essentially coincide with the predictions in the large- $N$ limit. We have also studied the $N=2$ case, but the exponents are unaltered within numerical uncertainties. This suggests that the exponents are independent of $N$ without $\mathrm{O}\left(1 / N^{2}\right)$ corrections, possibly due to the BPS nature of the supergravity modes. Furthermore, since the exponents cannot depend on the coupling constant $\lambda$, the same exponents should be valid in the M-theory regime, where we consider the large- $N$ limit with fixed $g_{s} \sim g_{\mathrm{YM}}^{2}$ and with the scaling $\left|t-t^{\prime}\right| \sim N\left|\tau-\tau^{\prime}\right|$ of the light-cone time (in the string unit), corresponding to the infinite momentum limit $P^{+}=N / g_{s} \rightarrow \infty$. For possible interpretations of the predicted exponents from the $11 \mathrm{di}-$ mensional viewpoint of the Matrix theory conjecture, we refer the reader to Ref. [10] in addition to [9]. More details of our analysis as well as results on other correlation functions shall be reported in a separate paper.

Acknowledgments. - We thank M. Terashi for his contribution at the early stage of this work. We are also grateful to O. Aharony and Y. Kikukawa for useful discussions and comments. Computations have been carried out on PC clusters at KEK and Yukawa Institute. The present work is supported in part by Grant-in-Aid for Scientific Research (No. 19340066 and 20540286 for J.N., No. 21740216 for Y.S. and No. 20340048 for T.Y.) from Japan Society for the Promotion of Science.

* Electronic address: masanori.hanada@weizmann.ac.il

$\dagger$ Electronic address: jnishi@post.kek.jp

¥ Electronic address: ysekino@v101.vaio.ne.jp

$\S$ Electronic address: tam@hep1.c.u-tokyo.ac.jp

[1] T. Banks, W. Fischler, S. H. Shenker and L. Susskind, Phys. Rev. D 55, 5112 (1997). For a comprehensive review of the Matrix theory, see, e.g., W. Taylor, Rev. Mod. Phys. 73, 419 (2001).

[2] E. Witten, Nucl. Phys. B 443, 85 (1995).

[3] Y. Okawa and T. Yoneya, Nucl. Phys. B538, 67 (1999); B541, 163 (1999).

[4] J. M. Maldacena, Adv. Theor. Math. Phys. 2, 231 (1998).

[5] S. S. Gubser, I. R. Klebanov and A. M. Polyakov, Phys. Lett. B 428, 105 (1998); E. Witten, Adv. Theor. Math. Phys. 2, 253 (1998).

[6] N. Itzhaki, J. M. Maldacena, J. Sonnenschein and S. Yankielowicz, Phys. Rev. D 58, 046004 (1998).

[7] A. Jevicki and T. Yoneya, Nucl. Phys. B 535, 335 (1998).

[8] D. N. Kabat and W. Taylor, Phys. Lett. B 426, 297 (1998); W. Taylor and M. Van Raamsdonk, JHEP 9904, 013 (1999); Nucl. Phys. B 558, 63 (1999).

[9] Y. Sekino and T. Yoneya, Nucl. Phys. B 570, 174 (2000); Y. Sekino, Nucl. Phys. B 602, 147 (2001)

[10] T. Yoneya, Classi. Quantum Gravity 17, 1307(2000).

[11] E. Witten, Nucl. Phys. 460, 335 (1996).

[12] M. Asano, Y. Sekino and T. Yoneya, Nucl. Phys. B 678, 197 (2004); M. Asano and Y. Sekino, Nucl. Phys. B 705, 33 (2005); M. Asano, JHEP 0412, 029 (2004).

[13] M. Hanada, J. Nishimura and S. Takeuchi, Phys. Rev. Lett. 99, 161602 (2007).

[14] K. N. Anagnostopoulos, M. Hanada, J. Nishimura and S. Takeuchi, Phys. Rev. Lett. 100, 021601 (2008); M. Hanada, Y. Hyakutake, J. Nishimura and S. Takeuchi, Phys. Rev. Lett. 102, 191602 (2009).

[15] See also Ref. [16] for Monte Carlo simulations based on the lattice regularization.

[16] S. Catterall and T. Wiseman, JHEP 0712, 104 (2007); Phys. Rev. D 78, 041502 (2008); arXiv:0909.4947 [hepth].

[17] M. Hanada, A. Miwa, J. Nishimura and S. Takeuchi, Phys. Rev. Lett. 102, 181602 (2009).

[18] See Ref. [19] for a numerical analysis based on the discrete light-cone quantization in the $(1+1)$-dimensional case.

[19] J. R. Hiller, S. S. Pinsky, N. Salwen and U. Trittmann, Phys. Lett. B 624, 105 (2005).

[20] M. A. Clark and A. D. Kennedy, Nucl. Phys. Proc. Suppl. 129, 850 (2004).

[21] P. Yi, Nucl. Phys. B505, 307 (1997); S. Sethi and M. Stern, Comm. Math. Phys. 194, 675 (1998); G. Moore, N. Nekrasov and S. Shatashvili, ibid 209, 77 (2000) and references therein. 